\title{
因HAD
}

ISSN-L: 2530-5115

DOI: http://doi.org/10.22585/hospdomic.v4i2.102

\section{Manejo farmacológico de la obstrucción intestinal maligna}

Pharmacological management of malignant bowel obstruction

Álvaro Fuentes Merlos', Rafael López-Bas Valero'

1. Hospital Universitario de San Juan de Alicante, Unidad de Hospitalización a Domicilio, Sant Joan d'Alacant, España.

Correspondencia/Correspondence

Álvaro Fuentes Merlos

afuentesmerlos@gmail.com

Recibido/Received

03.03.2020

Aceptado/Accepted

16.03.2020
Conflicto de Intereses/Competing interest Los autores declaran que no existe conflicto de interés alguno en el presente trabajo.

CÓMO CITAR ESTE TRABAJO | HOW TO CITE THIS PAPER

Fuentes A, Lopez-Bas R. Manejo farmacológico de la obstrucción intestinal maligna. Hosp Domic 2020;4(2):81-7. 


\section{RESUMEN}

La obstrucción intestinal maligna (OIM) es uno de los problemas más difíciles de manejar por las unidades de cuidados paliativos debido a la elevada carga de síntomas, a menudo recurrentes $y$, a veces, refractarios entre los pacientes con cáncer avanzado. La falta de estudios contundentes hace que la OIM se maneje empíricamente, existiendo una elevada variabilidad clínica y falta de consenso. Presentamos el caso clínico de un paciente ingresado a cargo de la Unidad de Hospitalización a Domicilio de San Juan de Alicante que presentaba un cuadro de oclusión de intestino delgado debido a implante mesentérico en cuadrante inferior derecho. Se realizó una combinación de agentes propulsores y antisecretivos que actuaron sinérgicamente reduciendo los síntomas gastrointestinales, y presentando una mejoría tanto en la calidad de vida como en el tiempo medio de supervivencia.

Palabras clave: Obstrucción Intestinal; Neoplasias Abdominales; Tratamiento Farmacológico; Cuidados Paliativos.

\section{ABSTRACT}

Malignant bowel obstruction (MBI) is one of the most difficult problems to manage by palliative care units because of the high burden of often recurrent and sometimes refractory symptoms among patients with advanced cancer. The lack of robust studies means that $\mathrm{MBI}$ is managed empirically, with high clinical variability and lack of consensus. We present the clinical case of a patient in charge of the Home Hospitalization Unit who presented a picture of small bowel occlusion due to mesenteric implant in the lower right quadrant. A combination of propellant and antisecretory agents were used, which acted synergistically to reduce gastrointestinal symptoms, and showed an improvement in both quality of life and average survival time.

Keywords: Intestinal Obstruction; Abdominal Neoplasms; Drug Therapy; Palliative Care. 


\section{INTRODUCCIÓN}

La prevalencia de la obstrucción intestinal maligna (OIM) en el mundo varía entre el 3 y el 15\%, dependiendo de las series revisadas. Entre el 5 y el $51 \%$ se presentan en pacientes con tumores de ovario, y del 10-28\%, en pacientes con tumores gastrointestinales. Otros tumores relacionados incluyen: gástrico (6-19\%), de páncreas (6-13\%), de vejiga (3-10\%) y de endometrio (3-11\%). El tiempo medio entre el diagnóstico del cáncer y la obstrucción intestinal maligna es de 14 meses, pero puede ser la primera manifestación de la enfermedad tumoral en el 2\% de los casos, y hasta en el $22 \%$, en series quirúrgicas. Esta enfermedad compromete el intestino delgado en el $61 \%$ de los casos; el intestino grueso, en el 33\%, y en el $20 \%$, ambos sitios, simultáneamente. Pueden observarse múltiples niveles de obstrucción hasta en el 80\% de los casos(1-3). Los criterios de OIM se basan en el compromiso intestinal debajo del ángulo de Treitz, asociado a neoplasia maligna y sin evidencia de curación(4).

La falta de estudios contundentes que sirvan para el desarrollo de protocolos concretos para el abordaje de esta entidad hace que la obstrucción intestinal se maneje empíricamente, existiendo marcadas variaciones en la práctica clínica(5). En este sentido, resulta complicado hacer estudios de buena calidad en este tipo de pacientes, dada la dificultad de un reclutamiento adecuado, una esperanza de vida corta y las dificultades éticas para el uso de grupos control. Además, la escasa respuesta a los tratamientos utilizados sumado al mal pronóstico, con un rápido deterioro del estado general, son factores que hacen de esta patología uno de los mayores retos para los servicios de cuidados paliativos, con un alto impacto sobre la calidad de vida del paciente y sus cuidadores(1-3, $6)$.

La OIM se trata de una patología compleja, que produce un alto grado de disconfort, debido a los síntomas ocasionados por las alteraciones mecánicas y funcionales a través de distintos mecanismos fisiopatológicos y que puede responder peor a las medidas convencionales de reposo intestinal, descompresión nasogástrica y líquidos intravenosos(7-9). Entre estos mecanismos se encuentran la oclusión del lumen intestinal, las alteraciones en la motilidad y la acumulación de secreciones. Estos mecanismos propuestos confirman la hipótesis de que una combinación de agentes propulsores, antiinflamatorios y antisecreceptores deberían producir mejorías clínicas en los pacientes con OIM(10). Esta combinación es conocida por los médicos de cuidados paliativos y puede resolver la obstrucción y los síntomas asociados. Medicamentos como la octreotide, que puede reducir la hiperemia, las secreciones intestinales y la presión intraluminal; la dexametasona dirigida a la reducción del edema de la pared intestinal; y la motilidad gastrointestinal que promueve la metoclopramida son ampliamente usados(7-9). Además, el amidotrizoato, medio de contraste hiperosmolar soluble en agua, diluye el contenido intestinal, disminuyendo el edema de pared, lo cual, a su vez, facilita el tránsito intestinal(3). En un metaanálisis con 15 estudios, Mercadante et al, evaluaron la eficacia del uso de octreotide en comparación con escopolamina butilbromuro. Los autores hallaron tasas de respuesta total de entre el 60-90\%, así como una mayor y más rápida eficacia con el uso de octreotide, al igual que una disminución en el uso de drenaje por sonda nasogástrica (SNG) e, incluso, en diferir su utilización(6,8).

Este tratamiento farmacológico, aplicado de forma temprana e intensiva, ha demostrado ser eficaz no sólo para controlar los síntomas gastrointestinales en pacientes inoperables sino también para revertir la obstrucción intestinal maligna, lo que permite una clara mejoría tanto en la calidad de vida como en la supervivencia de estos pacientes $(7,10)$. Aunque tales medicamentos se han utilizado con cierto éxito para paliar los síntomas, existen datos clínicos limitados sobre la efectividad de estos agentes, particularmente en combinación. En la tabla 1 resumen se presenta el manejo farmacológico detallado en una revisión de la literatura(3). 
Tabla 1: Manejo farmacológico de la obstrucción intestinal maligna

\begin{tabular}{|l|l|}
\hline \multicolumn{2}{|l|}{ Principales manifestaciones OIM y su tratamiento } \\
\hline Dolor nociceptivo somático continuo & Uso de analgésicos opioides potentes en dosis tituladas \\
\hline Dolor visceral cólico & $\begin{array}{l}\text { Butilbromuro de escopolamina 20-40 mg cada } 8 \text { horas (100mg } \\
\text { dosis máxima) }\end{array}$ \\
\hline $\begin{array}{l}\text { Aumento de secreciones } \\
\text { gastrointestinales }\end{array}$ & $\begin{array}{l}\text { Butilbromuro de escopolamina 20-40 mg cada } 8 \text { horas (100mg } \\
\text { dosis máxima) } \\
\text { Octreotida 100-300 } \mu \mathrm{mg} \text { cada } 8 \text { horas }\end{array}$ \\
\hline Náuseas y vómito & $\begin{array}{l}\text { Haloperidol 5-15mg /día } \\
\text { Metoclopramida } 40-240 \text { mg/día } \\
\text { Ondansetrón } 12-24 \text { mg/día } \\
\text { Olanzapina 2,5-20 mg/día }\end{array}$ \\
\hline Actividad inflamatoria local & \begin{tabular}{l} 
Dexametasona 6-16 mg/día \\
\hline
\end{tabular} \\
\hline
\end{tabular}

\section{DESARROLLO DE LA EXPERIENCIA}

Informamos sobre un caso clínico de un paciente de 70 años, con antecedentes de adenocarcinoma de colon derecho, diagnosticado en junio de 2014, que precisó de hemicolectomia derecha. Actualmente con progresión de enfermedad por metástasis pulmonares y peritoneales de nueva aparición. Es remitido por el servicio de Oncología Médica a la Unidad de Hospitalización a Domicilio de San Juan de Alicante para tratamiento en fase paliativa terminal. El paciente presenta cuadro de oclusión de intestino delgado debido a implante mesentérico en cuadrante inferior derecho, que fue tratado con perfusión de 4 mg de dexametasona y 30 mg de metoclopramida. 
Figura 1 y 2: Dilatación de asas con edema de pared
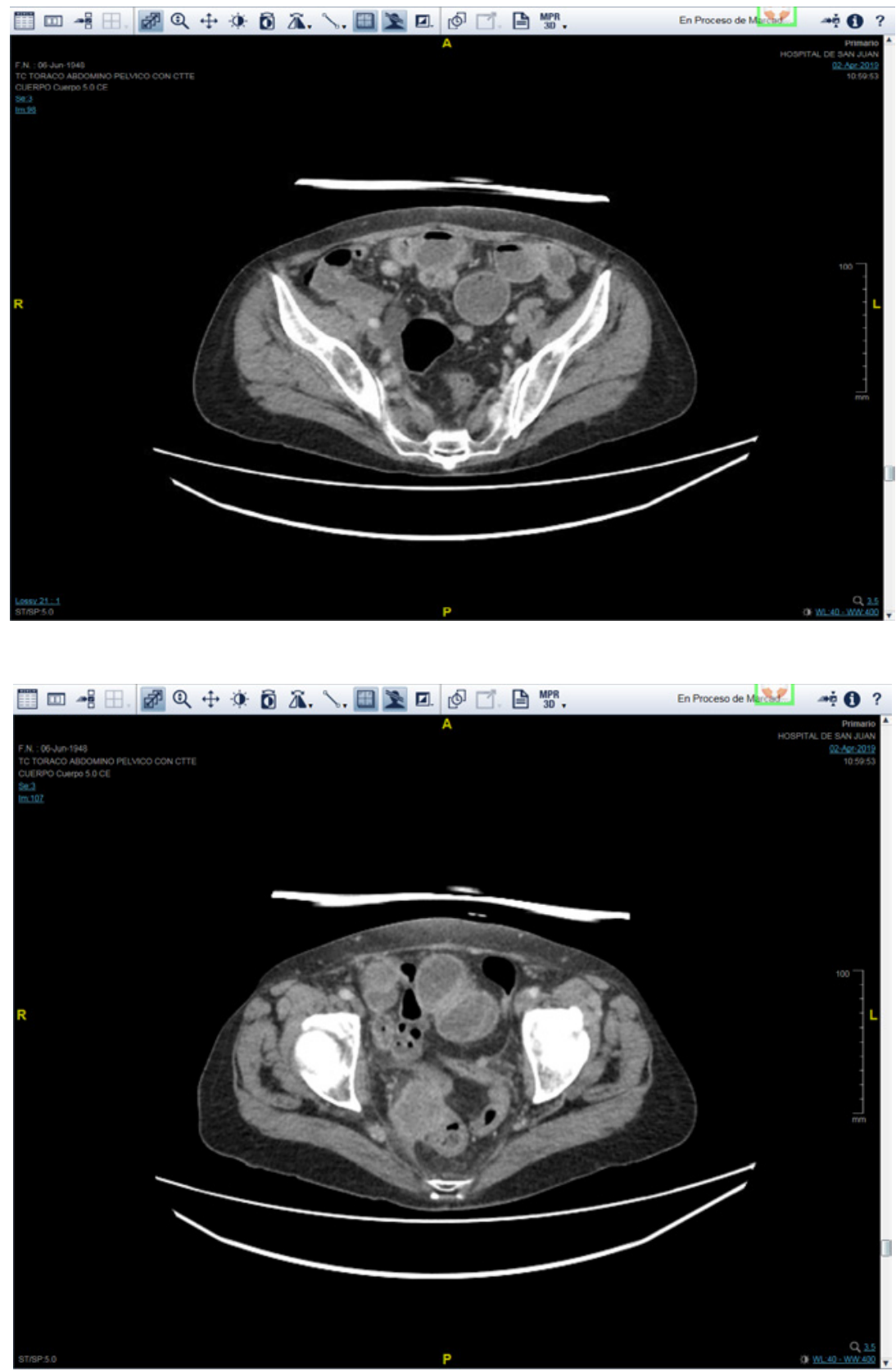
Por persistencia de sintomatología clínica de la OIM se decide iniciar en nuestra unidad perfusión de 1000cc SF a los que añadimos $12 \mathrm{mg}$ de ondasetron, $60 \mathrm{mg}$ de butilbromuro de escopolamina, $30 \mathrm{mg}$ de metoclorpamida y 50 microgramos de octreotide subcutánea a pasar en 24 horas. Se le añaden $500 \mathrm{ml}$ de suero glucosado y $12 \mathrm{mg}$ dexametasona por el reservorio. Además de 40 pantoprazol intravenoso.

A la valoración del día siguiente el paciente comienza a presentar aparición de ruidos hidroaéreos con expulsión de gases, manteniendo ingesta adecuada de líquidos. Sin nauseas y con una pequeña deposición. El paciente refiere además control adecuado del dolor con parches de fentanilo transdérmico. Al tercer día, se obtienen deposiciones suficientes, disminuyendo la distensión abdominal y el timpanismo a la exploración. Manteniendo dicha mejoría clínica durante 20 días.

A la tercera semana de comienzo de la perfusión, el paciente presenta empeoramiento clínico, con sensación nauseosa y vómitos fecaloides. Ante deterioro clínico y mínima respuesta al tratamiento agresivo de la oclusión intestinal, y dada la situación irreversible, se comenta dicho escenario con el paciente y los familiares, quien, de consenso tras entender y asumir etapa final de la vida, están conformes con adecuar el esfuerzo terapéutico y no colocar SNG. Al vigésimo tercer día de comenzar con la perfusión combinada de medicamentos, se acuerda con paciente y familiares iniciar sedación reglada añadiendo Midazolam 25 mg/día consiguiendo que el paciente quede dormido y en ausencia a estímulos (nivel 6 escala de sedación de Ramsay a las 6 horas) que se mantiene hasta el exitus del paciente en domicilio al vigésimo quinto día de iniciada la perfusión.

\section{DISCUSIÓN}

Controlar los síntomas y mejorar la calidad de vida son objetivos fundamentales de los cuidados paliativos. En el presente caso, la estrategia de tratamiento utilizada demostró ser altamente efectiva en un paciente con OIM inoperable, no sólo para reducir los síntomas gastrointestinales, es decir, los vómitos, sino también para prolongar el tiempo medio de supervivencia. Siendo en nuestro caso de 25 días desde iniciada la perfusión.

Este caso sugiere que la combinación de agentes propulsores y antisecretivos puede actuar sinérgicamente favoreciendo una rápida recuperación del tránsito intestinal sin inducir cólicos desagradables y mejorando la sintomatología del paciente. Este hecho se justificaría en que el mecanismo más importante de la OIM, en este contexto, es funcional y puede ser reversible incluso en pacientes con cáncer avanzado, si se inicia un tratamiento agresivo temprano antes de que la impactación fecal y el edema hagan irreversible la OIM.

\section{BIBLIOGRAFÍA}

1. Soriano A, Davis MP. Malignant bowel obstruction: Individualized treatment for patients near the end of life. Cleve Clin J Med. 2011;78(3):197-206. DOI: 10.3949/ccjm.78a.10052; PMID: 21364165

2. Tuca A, Guell E, Martínez-Losada E, Codorniu N. Malignant bowel obstruction in advanced cancer patients: epidemiology, management, and factors influencing spontaneous resolution. Cancer Res. 2012;4:159-69. DOI: 10.2147/CMAR.S29297; PMID: 22904637 
3. Cárdenas J, Agamez C, Parra S. Obstrucción intestinal maligna. Revisión de tema. Rev Colomb Cancerol. 2013;17(2):77-85.

4. Anthony T, Barón T, Mercadante S, Green S, Chi D, Cunning- ham J, et al. Report of the clinical protocol committee: development of randomized trials for malignant bowel obstruction. J Pain Symptom Manage. 2007;34(Suppl 1):S49-S59. DOI: 10.1016/j.jpainsymman.2007.04.011; PMID: 17544243

5. Feur DJ, Broadley KE, Sheperd JH, Barton DP. Surgery for the resolution of symptoms in malignant bowel obstruction in advanced gynecological and gas- trointestinal cancer. Cochrane Database Syst Rev 2000;(4):CD002764. DOI: 10.1002/14651858.CD002764; PMID: 11034757

6. Mercadante S, Casuccio A, Mangione S. Medical treatment for inoperable malignant bowel obstruction: A qualitative systematic review. J Pain Symptom Manage. 2007;33(2):217-23. DOI: 10.1016/j.jpainsymman.2006.06.014; PMID: 17280927

7. Mercadante S, Ferrera P, Villari P, Marrazzo A. Aggressive pharmacological treatment for reversing malignant bowel obstruction. J Pain Symptom Manage. 2004;28(4):412-6. DOI: 10.1016/j. jpainsymman.2004.01.007; PMID: 15471659

8. Mercadante S, Porzio G. Octreotide for malignant bowel obstruction: Twenty years after. Cri Rev Oncol Hematol. 2012;83(2):388-92. DOI: 10.1016/j.critrevonc.2011.12.006; PMID: 22277783

9. Mercadante S. Assessment and management of mechanical bowel obstruction. In: Portenoy RK, Bruera E, eds. Topics in Palliative Care. New York, USA: Oxford University Press; 1997. p.113-30.

10. Berger J, Lester P, Rodrigues L. Medical Therapy of Malignant Bowel Obstruction With Octreotide, Dexamethasone, and Metoclopramide. Am J Hosp Palliat Care. 2016;33(4):407-10. DOI: 10.1177/1049909115569047; PMID: 25646530 